\title{
Tradução, Adaptação e Evidências Iniciais de Validade da Magical Ideation Scale
}

\author{
Philipe Gomes Vieira ${ }^{1}$ \\ Anna Elisa de Villemor-Amaral \\ Giselle Pianowski \\ Universidade São Francisco
}

\begin{abstract}
RESUMO - Magical Ideation Scale (MIS) é uma escala de autorrelato que avalia pensamentos mágicos, fenômeno ligado à esquizofrenia. Este trabalho procurou adaptar a MIS à cultura brasileira, bem como investigar sua sensibilidade discriminativa a partir de um estudo de validade. Na etapa de adaptação, 283 sujeitos responderam a MIS, marcando palavras e itens incompreendidos. Posteriormente, administrou-se a escala a 70 sujeitos divididos em grupos: Pacientes e Não-pacientes. A comparação do desempenho de ambos foi obtida por meio do teste $t$ de Student, revelando diferença significativa e de acentuada magnitude, conforme d de Cohen. A correlação de Pearson entre o diagnóstico de esquizofrenia e a MIS evidenciou associação positiva de expressiva magnitude. Interpretam-se os resultados como evidências de validade para a MIS.
\end{abstract}

Palavras-chave: testes psicológicos, adaptação de teste, análise semântica, validade do teste, pensamento mágico

\section{Translation, Adaptation and Initial Validity Study of the Magical Ideation Scale}

\begin{abstract}
Magical Ideation Scale (MIS) is a self-report scale that assesses magical thinking, a phenomenon linked to schizophrenia. This study aimed to adapt the MIS to Brazilian culture, as well as investigate its discriminative sensitivity based on a validity study. In the adaptation stage, 283 subjects completed MIS, marking words and misunderstood items. Subsequently, the scale was administered to 70 subjects divided into groups: Patients and Non-patients. A comparison of the performance of both groups was realized using Student's t, which revealed significant differences, while Cohen's d showed a strong discrepancy between the two groups. The Pearson correlation between the diagnosis of schizophrenia and MIS showed a positive association of significant magnitude. The results are interpreted as evidence of validity for the MIS.
\end{abstract}

Keywords: psychological tests, test adaptation, semantic analysis, test validity, magical thinking

A Magical Ideation Scale (MIS), publicada em 1983 por Eckblad e Chapman, é uma escala de autorrelato, composta por 30 itens com possibilidade de resposta dicotômica, a qual propõe avaliar pensamentos mágicos. Conforme salientam os referidos autores, pensamento mágico é uma espécie de crença, por vezes tida como bizarra, que se distancia do pensamento coletivo, uma vez que está focada na possibilidade de eventos acontecerem sem que estes tenham relação causal apropriada aos padrões convencionais da cultura. Ainda para os criadores dessa escala, esse tipo de pensamento pode ser encontrado em quadros clínicos propensos à esquizofrenia, tal como o transtorno da personalidade esquizotípica (TPE).

De acordo com os Manuais Diagnóstico e Estatístico de Transtornos Mentais - DSM-IV (American Psychiatric Association [APA], 2000) e DSM-5 (APA, 2013)-, o TPE é caracterizado por um padrão persistente de comprometimento social e interpessoal, atrelado a distorções cognitivas e perceptuais, bem como a comportamentos excêntricos. Esse transtorno se inicia no começo da fase adulta e perdura ao longo da vida do sujeito, manifestando-se em diversos contextos. Embora os sujeitos acometidos por esse transtorno de personalidade não apresentem delírios, tampouco alucinações, frequentemente atribuem interpretações

1 Endereço para correspondência: Universidade São Francisco, R. Waldemar César da Silveira, 105, Vl. Cura D’Ars (SWIFT), Campinas, SP, Brasil. CEP. 13.045-510.E-mail: philipevieira@ymail.com incorretas acerca de eventos externos, acreditando que estes possuem significados peculiares para si próprios.

Clark (1990), Tonelli, Heyde, Alvarez e Raine (2009) e Skodol et al. (2011) ressaltam que o comportamento e/ou aparência do sujeito esquizotípico tendem a ser peculiares ou excêntricos, o que, por conseguinte, potencializa sua dificuldade acentuada de estabelecer contatos interpessoais íntimos, estando, ainda, tal dificuldade, associada com temores paranoides. Seu pensamento e discurso seguem a mesma direção, assumindo, muitas vezes, um caráter bizarro, que podem evidenciar-se vagos, estereotipados ou de cunho metafórico.

De modo geral, o sujeito com TPE costuma, ainda, apresentar comportamentos e crenças supersticiosos ligados a fenômenos paranormais, os quais não pertencem à sua subcultura. Assim sendo, revela acreditar que possui poderes especiais, como telepatia, clarividência e capacidade de ler a mente dos demais. Em contrapartida, é frequente a manifestação de ideações paranoides, especialmente em relação às intenções das pessoas que o rodeiam (APA, 2000; Clark, 1990; Eckblad \& Chapman, 1983; Millon, Grossman, Millon, Meagher, \& Ramnath, 2004; Tonelli, et al., 2009).

Parra (2008) corrobora a concepção de que comportamentos e experiências orientados à paranormalidade sejam característicos do funcionamento esquizotípico. O referido autor conduziu um estudo com o intuito de investigar padrões esquizotípicos em sujeitos que relatavam ser capazes 
de visualizar a aura dos demais. Em síntese, sua hipótese foi comprovada, sugerindo que aqueles que haviam relatado tal experiência evidenciavam maior nível de esquizotipia, bem como tendências dissociativas e propensão ao pensamento mágico $(z=4,50 ; p<0,001)$.

Em outro estudo, Parra (2010), partindo do pressuposto de que sujeitos que acreditavam em questões paranormais, superstições, psicocinesia, entre outras formas de pensamentos mágicos, estariam mais propensos à experiência alucinatória, propôs investigar a intensidade de sintomas atrelados à esquizotipia em sujeitos crentes na paranormalidade, dividindo-os em dois grupos: alucinadores e não alucinadores. Como resultado, obteve-se maior intensidade de imaginação auditiva $(z=3,21 ; p \leq 0,001)$ e visual $(z=3,22 ; p \leq 0,001)$ no grupo composto por sujeitos caracterizados como alucinadores, bem como elevados níveis de esquizotipia $(z$ $=4,20 ; p \leq 0,001)$.

Nesse sentido, o funcionamento esquizotípico pode ser compreendido como uma dimensão da personalidade que, em um continuum, apresenta comportamentos mais adaptados, em seu polo saudável e, por outro lado, propensão à manifestação de transtornos do tipo psicótico no polo mais patológico (Chapman, Edell, \& Chapman, 1980; Millon, et al., 2004; Parra, 2010). Cabe ressaltar, contudo, que aspectos sustentados culturalmente, como, por exemplo, crenças e/ou rituais religiosos, muito embora possam parecer exageradamente excêntricos, não podem ser considerados traços esquizotípicos. Desse modo, acreditar em vida após a morte, praticar rituais de voodoo, falar em línguas estranhas, ter pensamentos mágicos ligados às doenças e/ou saúde ou possuir um "sexto sentido" não devem servir para a classificação diagnóstica de TPE se tais aspectos estiverem intrinsecamente relacionados à subcultura do sujeito (APA, 2000; APA, 2013; Tonelli, et al., 2009).

Diante do exposto, Eckblad e Chapman (1983) relatam que os itens da MIS foram originalmente construídos de forma que o sujeito respondente pudesse interpretá-los com base em suas experiências pessoais, ao invés de ter que hipotetizar acontecimentos. Tais experiências contemplam "ideias de transmissão de pensamento, fenômenos psicocinéticos, premonição, astrologia, influência de espíritos, reencarnação, amuletos da sorte, e trocas de energias psíquicas entre pessoas" (Eckblad \& Chapman, 1983, p.215). Cabe ressaltar que muitos itens abordam experiências que seriam aceitáveis dependendo da subcultura do sujeito, o que, conforme discutido anteriormente, não poderia ser utilizado para subsidiar o diagnóstico de TPE ou demais transtornos do tipo psicótico. Contudo, alguns itens demonstram-se livres de tais influências, como, por exemplo, aqueles que fazem menção a presença de mensagens secretas e/ou subliminares no comportamento dos outros e na forma como objetos são organizados.

Eckblad e Chapman (1983) utilizaram esse instrumento para diferenciar universitários com e sem sintomas de propensão à psicose, tendo esse estudo demonstrado diferenças estatisticamente significativas entre os dois grupos para os sintomas de transmissão de pensamento $(M 1=1,0$; $\left.D P_{1}=2,1 ; M_{2}=0 ; D P=0 ; t=2,46 ; p<0,05\right)$, alucinações auditivas $\left(M_{1}=1,21 ; D P_{1}=2,4 ; M_{2}=0 ; D P=0 ; t=2,60 ; p\right.$
$<0,05)$; e crenças bizarras $\left(M_{1}=2,0 ; D P_{1}=3,2 ; M_{2}=0,2\right.$; $D P=0,80 ; t=2,81 ; p<0,05)$. Em outro estudo, apresentado por Chapman, Chapman, Kwapil, Eckblad e Zinser (1994), os sujeitos que compuseram a amostra evidenciaram alta magnitude de correlação entre o desempenho apresentado na MIS e traços esquizotípicos $(r=6,8 ; p<0,05)$, sugerindo significativa associação entre pensamento mágico e funcionamento esquizotípico.

A identificação de pensamentos mágicos e crenças bizarras, bem como o diagnóstico do TPE têm sido considerados de extrema importância para o tratamento clínico, uma vez que esse padrão pode ser estimado um fator de vulnerabilidade e propensão para o desenvolvimento da esquizofrenia, a qual ordinariamente tem seu início na fase adulta e possui um curso crônico (APA, 2000; APA, 2013; Tonelli, et al., 2009). Nesse sentido, o diagnóstico prévio do TPE é crucial para que ações preventivas e terapêuticas possam ser tomadas, minimizando o sofrimento causado por esse transtorno e, dessa forma, atenuando sua propensão à esquizofrenia.

Diante desse cenário, Eckblad e Chapman (1983) propuseram a construção da MIS com o intuito de auxiliar no diagnóstico de padrões inclinados à manifestação de sintomas psicóticos e desenvolvimento da esquizofrenia. Todavia, essa escala foi publicada nos Estados Unidos e, até o presente momento, não possui padronização para cultura brasileira. Em paralelo, verifica-se no Brasil uma escassez de instrumentos de medida capazes de identificar padrões esquizotípicos, tal como a MIS se mostra eficaz. Sendo assim, o presente trabalho tem como objetivo propor uma versão brasileira da MIS, verificando se sua tradução evidencia adequação semântica inteligível para essa população, bem como investigar a sensibilidade discriminativa da escala por meio de um estudo inicial de validade de critério.

\section{Método}

\section{Participantes}

Para o estudo de tradução e adaptação da escala, participaram 283 sujeitos, residentes no interior de Minas Gerais, com idades entre 18 e 68 anos $(M=28,3 ; D P=11,10)$, sendo $71 \%$ do sexo feminino $(f=203)$. No que diz respeito à formação, $50,9 \%$ da amostra era composta por universitários $(f=145), 36,5 \%$ por sujeitos do ensino médio $(f=104) \mathrm{e}$ $11,9 \%$ do ensino fundamental $(f=34)$. Com relação ao tipo de instituição, 43,9\% ( $f=125)$ eram alunos de instituições públicas e 55,4\% $(f=158)$, particulares.

Na sequência, para o estudo de evidências de validade, participaram, de maneira voluntária, 70 adultos, sendo $63,9 \%$ do sexo masculino, com idades variando entre 19 e 64 anos $(M=40,49 ; D P=11,042)$, em diferentes níveis de escolaridade, habitantes do interior de Minas Gerais. Os participantes foram divididos em dois grupos, pacientes e não-pacientes, e pareados no que concerne às características pessoais, como idade e escolaridade. $\mathrm{O}$ grupo de pacientes foi composto por 35 sujeitos que frequentavam, para consulta e acompanhamento, uma instituição especializada em saúde 
mental. Todos os pacientes faziam uso de medicação e eram atendidos por uma equipe multiprofissional composta por psicólogos, psiquiatras, terapeutas ocupacionais e enfermeiros. O critério de inclusão desse grupo era ter previamente recebido o diagnóstico nosográfico de esquizofrenia, sustentado a partir do código referente à Classificação Internacional de Doenças - CID-10 (OMS, 1993). É válido destacar que foi realizada uma pré-seleção, a partir dos registros médicos, para destacar sujeitos que apresentavam o diagnóstico adicional de deficiência intelectual, uma vez que tal condição poderia comprometer a utilização de uma escala de autorrelato. Todos os pacientes que compuseram a amostra se encontravam em um estágio avançado de tratamento psicológico e psiquiátrico, sendo, portanto, considerados estabilizados.

Em paralelo, o grupo não-pacientes foi representado por 35 sujeitos que não apresentavam histórico de comprometimento mental e/ou psicológico. Os participantes dessa amostra foram selecionados por meio de indicações de terceiros.

\section{Instrumento}

A MIS é uma escala de autorrelato construída para identificar a presença de pensamento mágico. Os itens propostos por Eckblad e Chapman (1983) contemplam

Tabela 1. Versão final dos itens traduzidos e adaptados da Magical Ideation Scale com os direcionamentos para apuração*

01. Algumas pessoas me fazem lembrar delas apenas ao pensarem em mim.

(Verdadeiro)

02. Já tive uma rápida sensação de que eu poderia não ser humano.

(Verdadeiro)

03. Às vezes tenho medo de pisar nas riscas das calçadas.

(Verdadeiro)

04. Eu acho que poderia aprender a ler a mente das pessoas se eu quisesse.

(Verdadeiro)

05. Horóscopos estão frequentemente certos para ser apenas coincidência.

(Verdadeiro)

06. Às vezes as coisas parecem estar em lugares diferentes quando eu chego em casa, mesmo sem ninguém ter estado lá.

07. Números como 13 e 7 não possuem poderes especiais.

(Verdadeiro)

08. Já tive, algumas vezes, a tola sensação de que um apresentador de TV ou rádio sabia que eu o estava escutando.

09. Eu já tive receio de que pessoas de outros planetas pudessem estar influenciando o que acontece na Terra.

10. O governo se recusa a nos contar a verdade sobre discos voadores.

(Verdadeiro)

(Verdadeiro)

11. Eu já tive a sensação de que havia mensagens para mim na maneira como as coisas estavam arranjadas, por

(Verdadeiro) exemplo, na vitrine de uma loja.

12. Eu nunca duvidei que meus sonhos fossem produtos da minha própria mente.

(Falso)

13. Amuletos da sorte não funcionam.

(Falso)

14. Eu já percebi sons nas minhas gravações que não estavam lá antes.

(Verdadeiro)

15. Às vezes parece que certos movimentos que estranhos fazem com as mãos me influenciam.

(Verdadeiro)

16. Eu quase nunca sonho com coisas antes que elas aconteçam.

(Falso)

17. Eu já tive a rápida sensação de que alguém havia sido substituído por um sósia.

(Verdadeiro)

18. Não é possível prejudicar alguém apenas tendo maus pensamentos sobre ele.

(Falso)

19. Já tive a sensação de ter algo mau perto de mim, embora eu não pudesse vê-lo.

20. Às vezes tenho a sensação de ganhar ou perder energia quando certas pessoas me olham ou me tocam.

(Verdadeiro)

21. Eu já tive a rápida impressão de que estranhos estivessem apaixonados por mim.

(Verdadeiro)

22. Eu nunca tive a impressão de que certos pensamentos meus na verdade pertenciam a outra pessoa.

(Falso)

23. Quando sou apresentado a estranhos, raramente fico me perguntando se já os tinha conhecido antes.

(Falso)

24. Se reencarnação fosse verdade, isso explicaria algumas experiências incomuns que eu tive.

(Verdadeiro)

25. Pessoas muitas vezes se comportam de um modo tão estranho que a gente se pergunta se elas não fariam parte de

(Verdadeiro) algum experimento.

26. Às vezes eu pratico pequenos rituais para me livrar de influências negativas.

(Verdadeiro)

27. Já tive a impressão de que eu poderia fazer alguma coisa acontecer apenas por pensar muito nela.

28. Eu já me perguntei se os espíritos dos mortos podem influenciar os vivos.

29. Algumas vezes já senti que uma palestra ou aula era dirigida especialmente a mim.

*Nota. Este material foi originalmente publicado em inglês como Eckblad, M., \& Chapman, L. J. (1983). Magical ideation as an indicator of schizotypy. Journal of Consulting \& Clinical Psychology, 51, 215-225. Copyright (C) 1983 pela American Psychological Association (APA). Traduzido, adaptado e reproduzido com permissão. A APA não é responsável pela precisão desta tradução. Esta tradução não pode ser reproduzida ou distribuida sem autorização prévia por escrito da APA 
sintomas compatíveis com o TPE e demais transtornos propensos à esquizofrenia. As sentenças devem ser analisadas e assinaladas pelo sujeito em uma escala dicotômica, do tipo verdadeiro ou falso.

Os itens traduzidos e adaptados da MIS bem como as direções para pontuação constam da Tabela 1, sob a autorização da American Psychological Association, que mantém os direitos autorais do instrumento. De acordo com a proposta de Eckblad e Chapman (1983), considera-se um ponto para cada item assinalado pelo sujeito conforme o esperado. Os pontos de corte propostos pelos autores supracitados são os escores brutos 19 e 20 para homens e mulheres, respectivamente.

\section{Procedimentos}

O presente estudo foi dividido em dois momentos. No primeiro, referente à tradução e adaptação da MIS, os dois primeiros autores deste artigo realizaram a tradução da escala, analisando a adequação semântica e escolha dos sinônimos na língua portuguesa. Em paralelo, uma segunda versão traduzida da escala foi obtida a partir da análise de uma tradutora profissional. Posteriormente, buscou-se sintetizar as duas por consenso entre os autores. A versão alcançada foi submetida à revisão ortográfica por uma especialista em gramática portuguesa, sem conhecimento prévio do instrumento em sua versão original.

$\mathrm{Na}$ sequência, o instrumento piloto foi submetido à backtranslation pela terceira autora do presente estudo, também não conhecedora da versão original da MIS, para verificar a similaridade da versão brasileira com a original. Com a adequação da tradução verificada em consenso com o grupo de pesquisadores e após pequenos ajustes, chegou-se à versão final utilizada no presente estudo.

A etapa seguinte do estudo foi administrar a MIS a uma amostra de sujeitos adultos de variadas faixas etárias e diversos graus de escolaridade, a fim de verificar a adequação da linguagem da versão final para a realidade brasileira. Todos os participantes, voluntários alcançados por meio de um processo de amostragem não aleatório, assinaram o Termo de Consentimento Livre e Esclarecido antes de responderem a MIS. Ao responderem, os sujeitos eram orientados a assinalar no verso da folha de respostas as palavras cujo significado não conheciam, bem como os itens que porventura não foram capazes de compreender, acrescentando observações quanto aos elementos que dificultaram a sua compreensão. Posteriormente, os dados foram lançados em uma planilha do Statistical Package for Social Sciences (SPSS) para a realização das análises estatísticas. Além de serem obtidas as estatísticas descritivas, procedeu-se à ANOVA a fim de identificar se havia diferenças estatisticamente significativas entre os grupos em função de sua respectiva escolaridade, bem como se buscou correlacionar o escore bruto da MIS com o diagnóstico prévio de esquizofrenia.

No segundo momento da pesquisa, referente ao estudo de validade, foi realizado contato com uma instituição que presta atendimento clínico psicológico e psiquiátrico, situada em uma cidade do interior de Minas Gerais a fim de se obter a autorização para o desenvolvimento da pesquisa.
Posteriormente, o projeto foi encaminhado ao comitê de ética da Universidade São Francisco e, após sua aprovação, deu-se início ao processo de coleta de dados.

Os sujeitos do grupo de Pacientes, diagnosticados previamente pelos psiquiatras da instituição como esquizofrênicos do tipo paranoide, que demonstraram interesse em compor a amostra, receberam o Termo de Consentimento Livre e Esclarecido (TCLE) explicando basicamente os objetivos da presente pesquisa, garantindo, ainda, a confidencialidade das informações coletadas. Cabe ressaltar que os psiquiatras da instituição possuíam ampla experiência em sua área de atuação e, para estabelecer o diagnóstico diferencial dos pacientes, realizavam entrevistas livres baseando-se nas diretrizes publicadas no manual diagnóstico CID-10 (OMS, 1993). O grupo não-paciente, composto por não pacientes, era representado por sujeitos sem histórico de busca por tratamento psicológico e/ou psiquiátrico.

Tabela 2. Frequência de palavras desconhecidas

\begin{tabular}{lccc}
\hline & $\boldsymbol{\%}$ & $\boldsymbol{f}$ & Total \\
\cline { 2 - 4 } Sósia & 4,2 & 12 & 283 \\
Riscas & 0,4 & 1 & 286 \\
Experimento & 1,8 & 5 & 283 \\
Ocasionalmente & 0,6 & 1 & 283 \\
Momentânea & 1,1 & 3 & 283 \\
Amuletos & 0,7 & 2 & 283 \\
\hline
\end{tabular}

\section{Resultados e Discussão}

Conforme mencionado anteriormente, o presente estudo se dividiu em dois momentos, tradução e adaptação, seguidos de um estudo inicial de validade. Dessa forma, os resultados serão apresentados de maneira sequencial, respeitando a ordem dos acontecimentos. Na etapa de tradução e adaptação, foram realizadas análises descritivas para as palavras que foram apontadas como desconhecidas, conforme a Tabela 2.

A análise de dados revelou seis palavras que foram pontuadas por pelo menos um dos participantes como desconhecida. Observa-se que a palavra que obteve a maior frequência de citação como desconhecida foi a palavra sósia, mencionada por $4,2 \%$ dos participantes. Pela análise, entendese que, dos itens traduzidos, poucas palavras apresentaram-se difíceis e para um grupo pequeno, considerado inexpressivo, visto que demonstra boa adequação para $95 \%$ da amostra estudada.

Em paralelo, as palavras apontadas como desconhecidas foram analisadas quanto ao nível de escolaridade, conforme as tabelas 3 e 4 .

As análises comparativas para os presentes dados são superficiais, visto o número reduzido de pessoas que apresentaram alguma dificuldade no reconhecimento de uma palavra usada na tradução da MIS. Sendo assim, com N máximo de 12 pessoas, para a comparação da palavra sósia entre grupos de acordo com os níveis de escolaridade, não há informações suficientes para gerar dados estatisticamente significativos. No entanto, as análises foram feitas no intuito de verificar se havia uma relação, já esperada, de que as palavras citadas como desconhecidas neste estudo tenham 
Tabela 3. Frequência de palavras desconhecidas por escolaridade

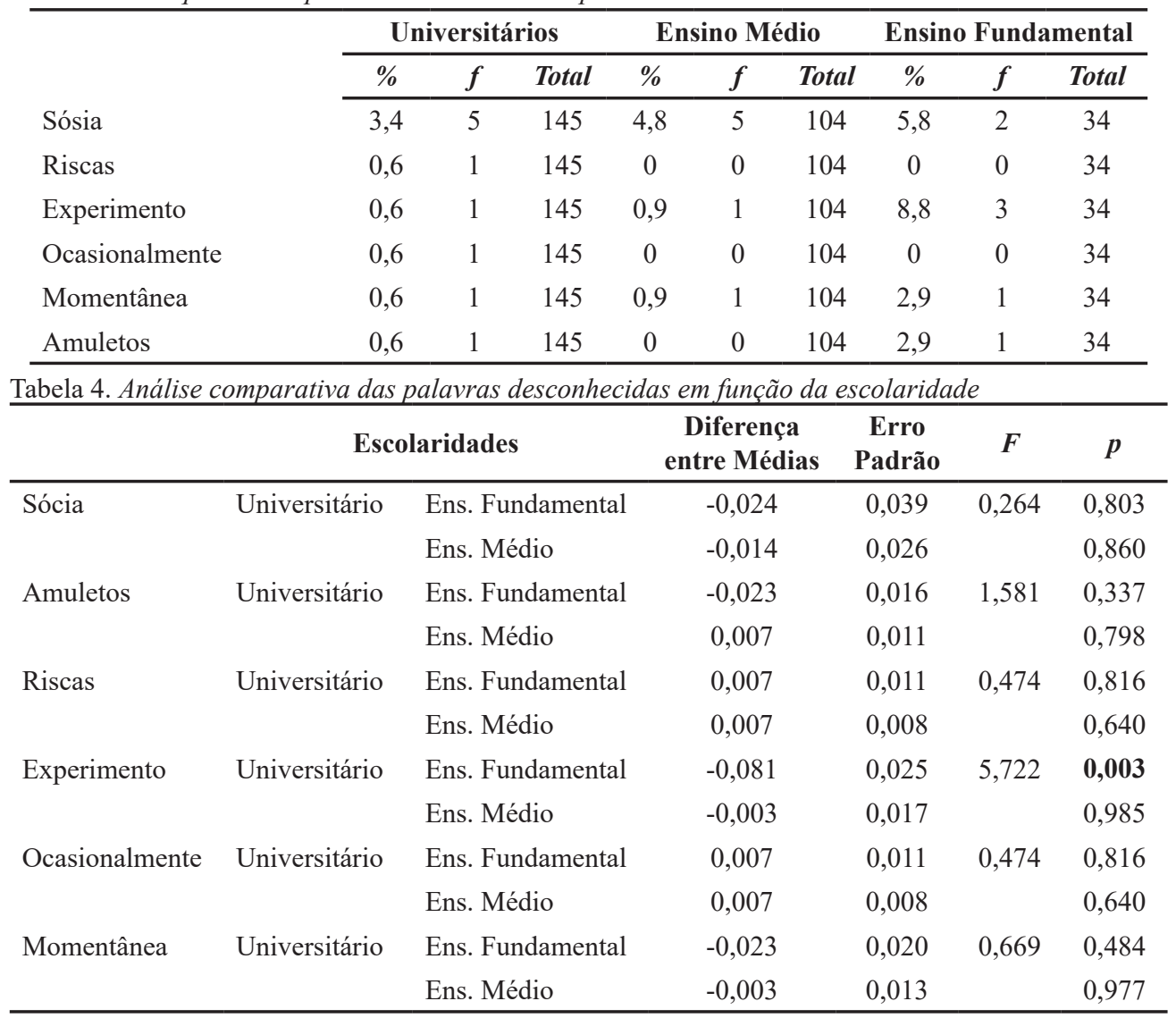

sido mencionadas prioritariamente no grupo com menor grau de escolaridade.

Os dados seguiram o esperado e demonstram que a palavra que teve maior incidência como desconhecida, a palavra sósia, apesar de não apresentar diferença significativa entre os grupos, apresentou uma tendência a diminuir sua citação como desconhecida em níveis de escolaridade mais altos. Esse argumento é confirmado com a palavra experimento, que, por meio da ANOVA, revelou diminuição estatisticamente significativa nos grupos com maiores níveis de escolarização.

Ademais das análises de palavras desconhecidas, foram realizadas análises para verificar os itens considerados de difícil compreensão pelos participantes. Ao se analisarem os itens apontados como não compreendidos, obteve-se um total de 22 itens. No entanto, 16 destes aparecem em menos de $1 \%$ dos protocolos coletados. Desse modo, optou-se por apresentar apenas os itens não compreendidos por mais de $1 \%$ da amostra, resultando em um total de seis itens, conforme apresentado na Tabela 5 .

Tabela 5. Itens não compreendidos

\begin{tabular}{lccc}
\hline & $\boldsymbol{f}$ & $\boldsymbol{\%}$ & Total \\
\cline { 2 - 4 } Item 1 & 29 & 10,2 & 283 \\
Item 14 & 8 & 2,8 & 283 \\
Item 15 & 5 & 1,8 & 283 \\
Item 16 & 9 & 3,2 & 283 \\
Item 17 & 5 & 1,8 & 283 \\
Item 24 & 5 & 1,8 & 283 \\
\hline
\end{tabular}

Observa-se que o item que mais apresentou-se difícil para os participantes foi o item 1 , incompreendido por mais de $10 \%$ da amostra e traduzido como Algumas pessoas me fazem pensar nelas apenas ao pensarem em mim. O restante dos itens mostrou-se bem compreendido por mais de $95 \%$ da amostra estudada, nos diferentes níveis de escolaridade. O segundo item notificado como não compreendido, o item 6 , apresentou-se difícil para pouco mais de $3 \%$ da amostra e foi traduzido como As vezes as coisas parecem estar em lugares diferentes quando eu chego em casa, mesmo sem ninguém ter estado lá.

Para maior avaliação da não compreensão dos itens citados na Tabela 5, foram também realizadas análises de frequência por nível de escolaridade. Tais análises são apresentadas na Tabela 6 .

No decorrer do processo de tradução, observou-se a necessidade de adaptação de alguns termos norte-americanos para o contexto brasileiro, buscando, no entanto, não comprometer a sensibilidade do item ao que pretende representar. Tal procedimento veio da constatação de que, por vezes, a tradução literal comprometia o entendimento do item e acabava não representando adequadamente o que a versão original sugeria. A organização dos itens adaptados para a versão final foi realizada como apresentado a seguir.

O primeiro item original da MIS, Some people can make me aware of them just by thinking about me, demonstrou-se, a priori, de difícil tradução, já que a expressão make me aware tem sua tradução como conscientizar ou trazer à consciência, o que, em contexto norte-americano explicita claramente a ideia de trazer à memória espontaneamente. No entanto, em 
Tabela 6. Itens não compreendidos por escolaridade

\begin{tabular}{cccccccccc}
\hline & \multicolumn{3}{c}{ Universitários } & \multicolumn{3}{c}{ Ensino Médio } & \multicolumn{3}{c}{$\begin{array}{c}\text { Ensino } \\
\text { fundamental }\end{array}$} \\
\cline { 2 - 11 } & \% & f & Total & \% & f & Total & $\%$ & f & Total \\
\cline { 2 - 11 } Item 01 & 13,1 & 19 & 145 & 6,7 & 7 & 104 & 8,8 & 3 & 34 \\
Item 14 & 2,7 & 4 & 145 & 2,8 & 3 & 104 & 2,9 & 1 & 34 \\
Item 15 & 2,7 & 4 & 145 & 0,9 & 1 & 104 & 0 & 0 & 34 \\
Item 16 & 3,4 & 5 & 145 & 3,8 & 4 & 104 & 0 & 0 & 34 \\
Item 17 & 0,7 & 1 & 145 & 0,9 & 1 & 104 & 2,9 & 1 & 34 \\
Item 24 & 0,7 & 1 & 145 & 0,9 & 2 & 104 & 5,8 & 2 & 34 \\
\hline
\end{tabular}

português, o termo conscientizar, não pareceu adequado para o entendimento do item. Dessa forma, optou-se pelos termos me fazem pensar, tornando a tradução mais adequada. Como resultado, o item ficou Algumas pessoas me fazem pensar nelas apenas ao pensarem em mim. Contudo, após a coleta de dados, percebeu-se a necessidade de se realizar pequenos ajustes, conforme apontamentos feitos pelos respondentes da versão piloto da escala, uma vez que esse item se mostrou confuso para $10,2 \%$ da amostra. Nesse sentido, tal item, após análise dos comentários realizados pelos respondentes e da avaliação dos juízes, foi editado para Algumas pessoas me fazem lembrar delas apenas ao pensarem em mim.

No segundo item, I have had the momentary feeling that I might not be human, a palavra momentary foi substituída por rápida, após a coleta de dados do presente estudo, pois, embora a frequência com que essa palavra fora assinalada como desconhecida tenha sido baixa, julga-se que essa modificação traz o item para um contexto mais coloquial. Dessa maneira, obteve-se o item Já tive uma rápida sensação de que eu poderia não ser humano.

No item 5, a expressão too often na frase Horoscopes are right too often for it to be a coincidence foi simplificada de muito frequentemente para frequentemente, uma vez que, na
Uma vez adaptada para a cultura brasileira, a MIS foi então administrada a 70 sujeitos adultos, os quais foram divididos em Pacientes e Não-pacientes. O desempenho dos participantes dos dois grupos foi comparado por meio do teste $t$ de Student, e a magnitude da diferença obtida a partir do $d$ de Cohen. Com base no diagnóstico nosográfico de esquizofrenia, critério externo utilizado no presente estudo, aventou-se a hipótese de que o grupo de Pacientes evidenciaria um escore bruto mais elevado quando comparado ao grupo Não-pacientes, o que pode ser confirmado, conforme apresentado na Tabela 7.

Os resultados apresentados na Tabela 7 revelam elevada discrepância acerca do desempenho de ambos os grupos, tendo em vista que o grupo de pacientes demonstrou maior média ([Pacientes: $M=18,71 ; D P=4,416]$; [Controle: $M$ $=4,74 ; D P=2,737])$. A elevada magnitude dessa diferença $(d=3,85 ; p=0,001)$ sugere que sujeitos que apresentam o diagnóstico de esquizofrenia têm maior probabilidade de pontuarem na MIS de maneira exacerbada, revelando, portanto, mais indícios de pensamentos mágicos.

Com o intuito de verificar se havia associação entre o escore bruto apresentado na MIS, que tem como objetivo avaliar a presença de pensamentos mágicos, com a presença

Tabela 7. Comparação do desempenho dos grupos na Magical Ideation Scale

\begin{tabular}{cccccccccc}
\hline & Grupo & N & Média & DP & EP & GL & $\boldsymbol{t}$ & $\boldsymbol{d}$ & $\boldsymbol{p}$ \\
\hline \multirow{2}{*}{ MIS } & Pacientes & 35 & 18,71 & 4,416 & 0,747 & 68 & & & \\
& Não-pacientes & 35 & 4,74 & 2,737 & 0,463 & 56,759 & 15,909 & 3,85 & 0,001 \\
& & & & & & & & \\
\hline
\end{tabular}

cultura brasileira, essa palavra já denota intensidade e, tentar enfatizar esse aspecto soaria como redundância. Assim sendo, a tradução resultou em Horóscopos estão frequentemente certos para ser apenas coincidência.

No item 8, I have occasionally had the silly feeling that a TV or radio broadcaster knew I was listening to him, as palavras occasionally e broadcaster, literalmente traduzidas como ocasionalmente e apresentador ou radiodifusor foram preteridas pelas expressões algumas vezes e pessoa, respectivamente. As traduções literais dessas palavras não atendem efetivamente a adequação coloquial para a cultura brasileira. A segunda palavra, por exemplo, quando traduzida, poderia ser radiodifusor, que, por sua vez, é totalmente incomum na forma de expressão do português. Diante do exposto, o item resultou em Já tive, algumas vezes, a tola sensação de que uma pessoa da TV ou do rádio sabia que eu a estava escutando. do diagnóstico de esquizofrenia, critério externo obtido pela avaliação dos psiquiatras da instituição de Saúde Mental, procedeu-se ao teste estatístico $r$ de Pearson. O resultado dessa correlação é apresentado na Tabela 8.

Os dados acima apresentados revelam associação de forte magnitude entre o escore bruto obtido na MIS e o diagnóstico nosográfico de esquizofrenia. Assim sendo, percebe-se associação positiva entre possuir o diagnóstico de esquizofrenia e apresentar elevada pontuação em tal escala. Diante do exposto, sujeitos esquizofrênicos expressam

Tabela 8. Correlação entre o escore bruto da MIS e o diagnóstico de esquizofrenia

\begin{tabular}{lcc}
\hline \multicolumn{1}{c}{ Variáveis } & $\boldsymbol{r}$ & $\boldsymbol{p}$ \\
\hline Escore bruto MIS versus & 0,88 & 0,001 \\
Diagnóstico nosográfico & & \\
\hline
\end{tabular}


maior propensão a pontuarem de maneira acentuada na MIS, corroborando a hipótese previamente aventada.

\section{Considerações Finais}

Os resultados sugerem que a MIS pode ser administrada a sujeitos adultos, com nível de escolaridade a partir do ensino fundamental. Quanto ao tipo da instituição de ensino, se pública ou particular, percebe-se adequação do instrumento independente de tal questão.

Em síntese, após analisar os dados coletados, tornase evidente que a escolha dos sinônimos utilizados para tradução dos itens da MIS demonstra-se adequada e inteligível para a amostra pesquisada. É possível, ainda, interpretar os resultados aqui apresentados como evidências iniciais de validade para a MIS, uma vez que a escala, após ter sido traduzida para o português, foi sensível para discriminar pacientes com o diagnóstico de esquizofrenia dos demais sujeitos que não apresentavam histórico de comprometimentos intrapsíquicos severos.

Cabe ressaltar que o presente estudo possui limitações a serem sanadas em eventuais pesquisas futuras. $\mathrm{O}$ aumento do número de sujeitos do sexo feminino na amostra geral investigada quando comparado ao total de sujeitos do sexo masculino ilustra uma delas. Outro ponto a ser considerado é a realização de estudos considerando não apenas a variável sexo, mas a identidade de gênero, a fim de ampliar as possibilidades de utilização da escala. Ademais, acredita-se que seja importante buscar comparar o desempenho de mais grupos em relação aos que foram aqui estudados, como, por exemplo, pacientes com propensão à esquizofrenia e não apenas os que já possuem tal diagnóstico.

Em suma, considera-se a versão utilizada da MIS adaptada para a cultura brasileira, necessitando, contudo, que estudos psicométricos contemplando a análise da fidedignidade por meio da consistência interna entre os itens, bem como a obtenção de valores descritivos ampliados que sirvam para a consolidação de expectativas normativas, sejam conduzidos na sequência para possibilitar sua utilização em contexto nacional.

\section{Referências}

American Psychiatric Association. (2000). Diagnostic and statistical manual of mental disorders (4th. Edition, Text. Rev.). Washington, D.C.: Author.

American Psychiatric Association. (2013). Diagnostic and statistical manual of mental disorders (5th. Edition). Arlington, VA: Author.

Chapman, L. J., Edell, E. W., \& Chapman, J. P. (1980). Physical anhedonia, perceptual aberration and psychosis proneness. Schizophrenia Bulletin, 6, 639-653.

Chapman, L. J., Chapman, J. P., Kwapil, T. R., Eckblad, M., \& Zinser, M. C. (1994). Putatively psychosis-prone subjects 10 years later. Journal of Abnormal Psychology, 103, 171-183.

Clark, L. A. (1990). Toward a consensual set of symptom clusters for assessment of personality disorder. In C. D. Spielberger (Org.), Advances in personality assessment (Vol. 8.) New Jersey: LEA.
Eckblad, M., \& Chapman, L. J. (1983). Magical ideation as an indicator of schizotypy. Journal of Consulting \& Clinical Psychology, 51, 215-225.

Millon, T., Grossman, S., Millon, C., Meagher, S., \& Ramnath, R. (2004). Personality disorders in modern life (2nd ed.) New Jersey: Wiley.

Organização Mundial de Saúde. (1993). Classificação de transtornos mentais e de comportamentos da CID-10: Descrições clínicas e diretrizes diagnósticas. Porto Alegre: Artmed.

Parra, A. (2008). La "visión del aura" como experiencia alucinatoria em individuos no-clinicos. Psico-USF, 13(2), 277-286.

Parra, A. (2010). Indicadores de propensión a la esquizotipia entre individuos creyentes en lo paranormal: Examinando la intensidad de la imaginería, esquizotipia y experiencias alucinatorias. Psicologia: Teoria e Prática, 12(3), 78-94.

Skodol, A. E., Clark, L. A., Bender, D. S., Krueger, R. F., Morey, L. C., Verheul, R., ... Oldham, J. M. (2011). Proposed changes in personality and personality disorder assessment and diagnosis for DSM-5, Part I: Description and rationale. Personality Disorders: Theory, Research, and Treatment, 2, 4-22.

Tonelli, H., Heyde, M., Alvarez, C., \& Raine, A. (2009). Versão em português do Questionário da Personalidade Esquizotípica SPQ. Revista Psiquiatria Clínica, 36(1), 34-37.

Recebido em 28.08.2014

Primeira decisão editorial em 09.02.2016

Versão final em 11.09.2016

Aceito em 11.09.2016 